\title{
Novel Stable Walking for Humanoid Robot Using Particle Swarm Optimization Algorithm
}

\author{
Tran Thien Huan \\ HCMC University of Technology and Education \\ Viet Nam
}

\begin{abstract}
This paper describes a novel walking gait generation algorithm based on inverse kinematics for a biped robot. The proposed algorithm uses the PSO (Particle Swarm Optimization) algorithm in order to find optimized values for the five walking algorithm parameters. The proposed experiment approach is tested on a small-sized humanoid robot in the DCSELAB, VNUHCM. Simulation and experimental results reveal that using the PSO algorithm with an efficient fitness function can significantly reduce learning time. Moreover, a considerable fast walking speed is achieved.
\end{abstract}

Keywords-PSO; walking robot; learning time; walking speed

\section{INTRODUCTION}

Biped robot locomotion is an exciting challenge for many researchers. In the recent years, a significant body of research was performed in this area. Early biped walking of robots involved static walking with a very low walking speed [1-2]. Hereby, the projected point of center of mass (COM) onto the ground always falls within the supporting polygon that is made by two feet. During the static walking, the robot can stop the walking motion any time without falling down. The disadvantage of static walking is that the motion is too slow and wide for shifting the COM. Researchers thus began to focus on dynamic walking of biped robots [3]. This approach is much faster than static balance. However, during dynamic walking, a biped robot may fall down from disturbances and cannot stop the walking motion suddenly. Hence, the notion of ZMP (Zero Moment Point) was introduced in order to control inertial forces [4-5]. Recently, lots of studies focused in the improvement the efficiency of the biped gait generation problem. Shaffi in [6] introduced the robust humanoid walking using truncated Fourier series gait generator. New methods using intelligent algorithms applied for biped gait generation development, such as bee colony optimization algorithm [7], TS fuzzy controller [8], evolutionary computing [9]. Especially, the potential of particle swarm optimization (PSO) has not yet been adequately applied in biped gait generation optimization.

To overcome this gap, this paper proposesa PSO-based biped gait generator, belongs to the dynamic balance methods, and utilizes ZMP criteria to maintain the stability of a biped robot. The outcomes of our simulations indicate that using the PSO to adjust biped walking parameters leads to a considerable fast walking speed with a negligible learning time.

\author{
Ho Pham Huy Anh \\ FEEE, HCM University of Technology, \\ VNU-HCM, Viet Nam
}

\section{Proposed Gait Generation Method}

The aim of inverse kinematics is to convert information in the Cartesian space to joint space. Hence, the derivation of the rules obtained from inverse kinematics will be presented. The most 5 important parameters of biped walking include s- step length [m], h- leg displacement [m], H- height of swing ankle [m], n- hip displacement [m] and T- step period [s]. Fig. 1 shows these 5 gait biped parameters.

As shown in Fig. 1, based on such 5 principal parameters, we determine the $\mathrm{x}-\mathrm{y}-\mathrm{z}$ coordinate of the biped's hip $P_{6}=\left[P_{6 x}, P_{6 y}, P_{6 z}\right]$, swing ankle $P_{12}=\left[P_{12 x}, P_{12 y}, P_{12 z}\right]$ and stance ankle $P_{0}=\left[P_{0 x}, P_{0 y}, P_{0 z}\right]$. Furthermore, $\mathrm{d}_{1}$ (length of shank) refers to the distance between the ankle pitch axis and knee pitch axis, while $\mathrm{d}_{2}$ (length of thigh) refers to the distance between the hip pitch axis and knee pitch axis, respectively.

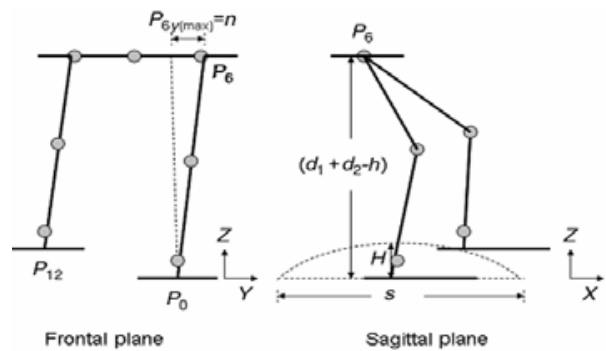

FIGURE I. FIVE PRINCIPAL GAIT GENERATION PARAMETERS OF BIPED.

The rules obtained from inverse kinematics require the inputs of Cartesian coordinates of the hip and swing ankle of the robot, which are functions of time since these values should be changing according to the system timer. Fig. 2 shows the step timeline which includes bring the back swing leg to the forward position.

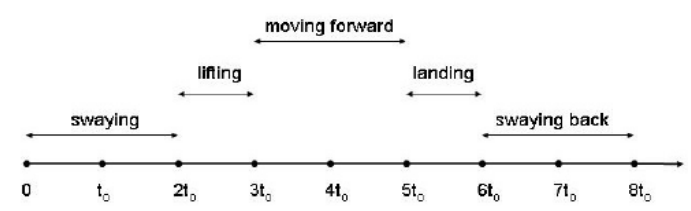

FIGURE II. TIMELINE OF A STEP TO BRING THE BACK LEG FORWARD.

\section{A. Design of Left Ankle Trajectories}

Inverse kinematics of biped gives $P_{0 x}$ being calculated as follows, 


$$
P_{0 x}(t)=\left(\frac{s}{2}\right) \cdot \sin \left[\frac{\pi}{T} \cdot\left(t-\frac{T}{2}\right)\right] \cdot[u(t-2 T)-u(t-T)]
$$

The same way, $P_{0 y}$ trajectory is calculated as follows,

$$
P_{0 y}= \begin{cases}0 & \text { if } 0 \leq t<T \\ w & \text { if } T \leq t<2 T\end{cases}
$$

In which $w$ the distance between two legs.

Finally, the $P_{0 z}$ trajectory is calculated as follows,

$$
P_{0 z}(t)=(H) \cdot \sin \left[\pi \cdot\left(\frac{P_{0 x}(t)}{s}+0.5\right) \cdot[u(t-2 T)-u(t-T)]\right]_{(3)}
$$

with $u(t)= \begin{cases}1 & \text { if } t \geq 0 \\ 0 & \text { if } t<0\end{cases}$

B. Design of Right Ankle Trajectories $P_{12}=\left[P_{12 x}, P_{12 y}, P_{12 z}\right]$

Inverse kinematics of biped gives $P_{12 x}$ being calculated as follows,

$$
P_{12 x}(t)=\left(\frac{s}{2}\right) \cdot \sin \left[\frac{\pi}{T} \cdot\left(t-\frac{T}{2}\right)\right] \cdot[u(t)-u(t-T)]
$$

The same way, $P_{12 y}$ trajectory is calculated as follows,

$$
P_{12 y}(t)=-w \cdot[u(t)-u(t-T)]_{(5)}
$$

Finally, the $P_{12 z}$ trajectory is calculated as follows,

$$
P_{12 z}(t)=H \cdot \sin \left[\pi \cdot\left(\frac{P_{12 x(t)}}{s}+0.5\right)\right] \cdot[u(t)-u(t-T)]
$$

with $u(t)= \begin{cases}1 & \text { if } t \geq 0 \\ 0 & \text { if } t<0\end{cases}$

\section{Design of Hip Trajectories $P_{6}=\left[P_{6 x}, P_{6 y}, P_{6 z}\right]$}

The same way, based on the inverse kinematics of biped,the $P_{6 x}, P_{6 y}, P_{6 z}$ trajectory can be determined as follows,

$$
\begin{gathered}
P_{6 x}(t)=\left(\frac{s}{4}\right) \cdot \sin \left[\frac{\pi}{t}\left(\tau-\frac{T}{2}\right)\right]_{(7)} \\
P_{6 y}(t)=P_{6 y_{-} \text {first_half_cycle }}(u(t)-u(t-T)) \\
-P_{6 y_{-} \text {first_half_cycle }}(u(t-T)-u(t-2 T))
\end{gathered}
$$

with $\tau= \begin{cases}t & \text { if } 0 \leq t<T \\ t-T & \text { if } T \leq t \leq 2 T\end{cases}$

$P_{0 z}(t)=(H) \cdot \sin \left[\pi \cdot\left(\frac{P_{0 x}(t)}{s}+0.5\right)\right] \cdot[u(t-2 T)-u(t-T)]$

with $u(t)= \begin{cases}1 & \text { if } t \geq 0 \\ 0 & \text { if } t<0\end{cases}$

Finally, all of 12 rotation joint angles' trajectories of the left and right leg of biped during two cycles can be determined based on P0-P6-P12 co-ordinate values using inverse kinematics.

\section{PSO-BASED GAIT GENERATION PARAMETERS OPTIMIZATION}

In PSO, a particle is defined as a moving point in hyperspace. For each particle, at the current time step, a record is kept of the position, velocity, and the best position found in the search space so far. Instead of using evolutionary operators such as mutation and crossover, to manipulate algorithms, for a $d$-variable optimization problem, a flock of particles are put into the $d$-dimensional search space with randomly chosen velocities and positions knowing their best values so far $\left(P_{\text {best }}\right)$ and the position in the $d$-dimensional space. The velocity of each particle, adjusted according to its own flying experience and the other particle's flying experience. For example, the $i$ th particle is represented as $x_{i}=\left(x_{i, 1}, x_{i, 2}, \ldots, x_{i, \mathrm{~d}}\right)$ in the $d$ dimensional space. The best previous position of the $i$-th particle is recorded and represented as:

$$
\text { Pbest }_{i}=\left(\text { Pbest }_{i, 1} \text {, Pbest }_{i, 2}, \ldots, \text { Pbest }_{i, \mathrm{~d}}\right)_{(10)}
$$

The index of best particle among all of the particles in the group in the $d$-dimensional space is gbest $_{d}$. The velocity for particle $i$ is represented as $v_{i}=\left(v_{i, 1}, v_{i, 2}, \ldots, v_{i, \mathrm{~d}}\right)$. The modified velocity and position of each particle can be calculated using the current velocity and the distance from Pbest ${ }_{i, \mathrm{~d}}$ to gbest $_{\mathrm{d}}$ as shown in the formulas:

$$
\left\{\begin{array}{l}
v_{i, m}^{(t+1)}=w v_{i, m}^{(t)}+c_{1} \text { Rand }() \cdot\left[\text { Pbest }_{i, m}-x_{i, m}^{(t)}\right]+c_{2} \text { Rand }() \cdot\left[\text { gbest }_{m}-x_{i, m}^{(t)}\right] \\
x_{i, m}^{(t+1)}=x_{i, m}^{(t)}+v_{i, m}^{(t+1)}, \quad i=1,2, \ldots, n ; m=1,2, \ldots, d
\end{array}\right.
$$

The evolution procedure of PSO Algorithms is shown in Fig. 3. 


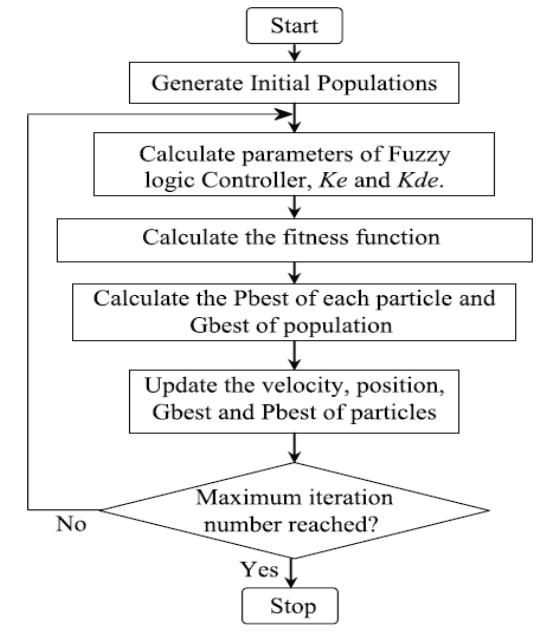

FIGURE III. EVOLUTION PROCEDURE OF PSO ALGORITHMS

After the fitness function has been calculated, the fitness value and the number of the generation determine whether or not the evolution procedure is stopped. Thus it calculates the Pbest of each particle and Gbest of population. The updated velocity and position, gbest and pbest of particles, give a new best position.

\section{A. PSO-based Gait Generation Parameters Optimization}

The PSO algorithm was selected because it can solve problems with less population density and less iteration in comparison with similar algorithms like GA. To make PSO convergence faster, the parameters is limited as presented in Table.1:

TABLE I. BIPED PRINCIPAL PARAMETERS’ LiMITS.

\begin{tabular}{|c|c|c|}
\hline Joint name & Lower bound & Uper bound \\
\hline HipHeight & $11 \mathrm{~cm}$ & $25 \mathrm{~cm}$ \\
\hline HipOffset & $1 \mathrm{~cm}$ & $10 \mathrm{~cm}$ \\
\hline Max AnkleHeight & $1 \mathrm{~mm}$ & $4 \mathrm{~cm}$ \\
\hline Steps & 3 & 14 \\
\hline GaitLength & $1 \mathrm{~cm}$ & $30 \mathrm{~cm}$ \\
\hline
\end{tabular}

As to reach an effective fitness function, the support area and zero momentum point ZMP were utilized. By means of these concepts, the biped stability can be measured effectively. In order to achieve a minimal deviation of ZMP and COM, the following function was used as the fitness function:

$$
\sum_{i=1}^{n}\left|\vec{Z}_{i}-\vec{C}_{i}\right|^{2}
$$

where $\mathrm{Zi}$ is $\mathrm{ZMP}$, $\mathrm{Ci}$ stands for the center of support area at cycle $i$, and $n$ shows the number of simulation cycles. On the other hand, it is clear that the best case is when the robot does not move (fitness $=0$ ). Therefore, another fitness function to combine with this function is required.

$$
\sum_{i=1}^{n-1}\left|\vec{C}_{i}-\vec{C}_{i+1}\right|^{2}
$$

By maximizing this function, the robot will move a longer path in a specific time (ncycles). In summary, the final formula of proposed fitness function which tries to minimize Eq. 13 and maximize the Eq. 14 as follows:

$$
f=\lambda \sum_{i=1}^{n}\left|\vec{Z}_{i}-\vec{C}_{i}\right|^{2}+\frac{1-\lambda}{\sum_{i=1}^{n-1}\left|\vec{C}_{i}-\vec{C}_{i+1}\right|^{2}}
$$

In this equation, the weighting coefficient $\lambda(0<\lambda \leq 1)$ can be used to choose the priority between stability (increase $\lambda$ ) and speed (decrease $\lambda$ ).

\section{SiMULATION AND EXPERIMENTAL RESUlTS}

The simulation and experiment approach is tested on a small-sized biped robot in the DCSELAB, VNU-HCM. In order to find the appropriate value for $\lambda$ parameter of the fitness function in Eq. 14, by choosing $\lambda=0.6$,biped achieves a fast and stable walking, and this value was used during PSO runs. Table 2 shows the PSO algorithms parameters. Table 3 shows optimized values of biped parameters.

TABLE II. THE PSO ALGORITHMS PARAMETERS.

\begin{tabular}{|c|c|c|c|c|}
\hline No. of Iterations & No. of Particles & Inertia & Particle Increment & Global Increment \\
\hline 30 & 20 & 0.98 & 0.01 & 0.01 \\
\hline
\end{tabular}

TABLE III. OPTIMIZED VALUES OF BIPED PRINCIPAL PARAMETERS.

\begin{tabular}{|c|c|c|c|c|}
\hline GaitLength & No. of steps & MaxAnkleHeight & HipOffet & HipHeight \\
\hline $19.3 \mathrm{~cm}$ & 8 & $3.0 \mathrm{~cm}$ & $8.3 \mathrm{~cm}$ & $17.9 \mathrm{~cm}$ \\
\hline
\end{tabular}

Fig. 4 illustrates the 2D-walking of biped gait generation in one cycleusing the PSO-based optimal parameters. Fig. 5 and Fig. 6 represent the rotation joint angles' trajectories of left and right leg of biped during 2 cycles using optimal parameters.

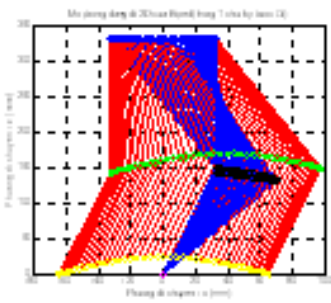

FIGURE IV. 2D-WALKING GAIT GENERATION OF BIPED IN ONE CYCLE.

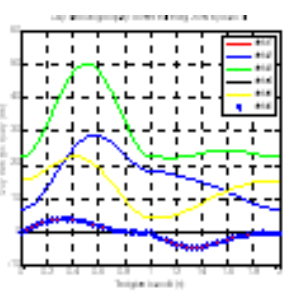

FIGURE V. ROTATION JOINT ANGLES’ TRAJECTORIES OF LEFT LEG IN TWO CYCLES USING OPTIMAL PARAMETERS. 


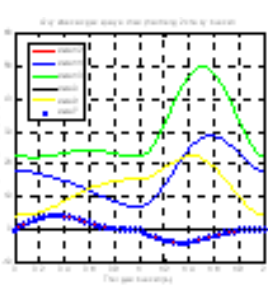

FIGURE VI. ROTATION JOINT ANGLES’ TRAJECTORIES OF RIGHT LEG IN TWO CYCLES USING OPTIMAL PARAMETERS.

These figures prove an excellent simulation and experiment results, which confirm the performance and the effectiveness of the PSO-based biped gait generation parameters optimization. Continually, Fig. 7-9 represent the good performance response of biped walking through the hip and ankles trajectories in $\mathrm{X}-\mathrm{Y}$ and $\mathrm{Z}$ direction using PSObased optimal parameters.

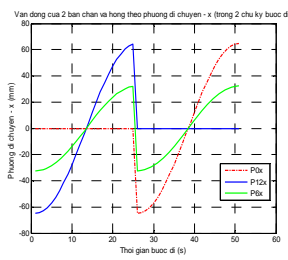

FIGURE VII. HIP AND ANKLES TRAJECTORIES IN X-DIRECTION USING OPTIMAL PARAMETERS.

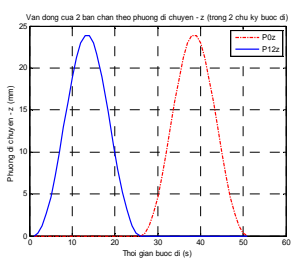

FIGURE VIII. TWO ANKLES TRAJECTORIES IN Z-DIRECTION.

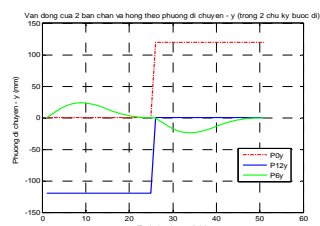

FIGURE IX. HIP AND ANKLES TRAJECTORIES IN Y-DIRECTION FROM OPTIMAL PARAMETERS.

\section{CONCLUSIONS}

This paper introduces a dynamic balance walking algorithm using the PSO algorithm. The inverse kinetics was used to estimate the position of each biped joint angle. Then, the PSO optimization algorithm was used to find the best values for gait biped parameters which generate the least deviation of ZMP from the biped support area. Simulation and experimental results reveal that using the PSO algorithm with an efficient fitness function can significantly reduce learning time.

\section{ACKNOWLEDGEMENTS}

This paper was supported by HCMUTE and VNU-HCM, Viet Nam.

\section{REFERENCES}

[1] W. T. Miller III, Real-time neural network control of a biped walking robot. IEEE Control Systems Magazine. 14(1): pp. 41-48 (1994)

[2] C. L. Shih,Ascending and descending stairs for a biped robot. IEEE Transactions on Systems, Man, and Cybernetics. 29(3): pp. 255-268 (1999)

[3] S. Kajita, F. Kanehiro, K. Kaneko, K. Fujiwara, K. Harada, K. Yokoi, H. Hirukawa,Biped walkingpattern generation by using preview control of Zero-Moment Point. Proceedings of the IEEE internationalconference on robotics and automation, Taipei, Taiwan, pp. 14-19 Sep. 2003

[4] M.Vukobratovic, et al.,Biped locomotion. Springer-Verlag (1990)

[5] J. Mrozowski, J. Awrejcewicz, Analysis of stability of the human gait, Journal of Theoreticaland Applied Mechanics, vol. 45, no. 1, pp. 91-98, 2007.

[6] N. Shafii, L.P. Reis, N. Lau,Biped Walking using Coronal and Sagittal Movements based on TruncatedFourier Series, RoboCup-2010: Robot Soccer World Cup XIII, Springer LNAI / LNCS, Vol. 6556, pp.324-335, 2011.

[7] E. Yazdi, V. Azizi, and A. T. Haghighat, "Evolution of biped locomotion using bees algorithm, based on truncated Fourier series," in Proceedings of the World Congress on Engineering and Computer Science, pp. 378-382, 2010.

[8] Y. Farzaneh, A. Akbarzadeh, A. Akbaria, Online bioinspired trajectory generation of seven-link biped robot based on T-S fuzzy system, Applied Soft Computing, 2013.

[9] D. Gong, J. Yan, and G. Zuo, "A review of gait optimization based on evolutionary computation," Applied Computational Intelligence and Soft Computing, vol. 2010, Article ID 413179, 12 pages, 2010. 